\title{
miRNA expression profile in abnormally invasive placenta: accreta, increta and percreta cases
}

\author{
Angelika Timofeeva \\ FSBI «National Medical \\ Research Center For Obstetrics, \\ Gynecology \\ And Perinatology Named After \\ Academician V.I.Kulakov» \\ Ministry of Healthcare of the Russian \\ Federation, Moscow, Russia \\ avtimofeeva28@gmail.com \\ Ivan Fedorov \\ FSBI «National Medical \\ Research Center For Obstetrics, \\ Gynecology \\ And Perinatology Named After \\ Academician V.I.Kulakov» \\ Ministry of Healthcare of the Russian \\ Federation, Moscow, Russia \\ i_fedorov@oparina4.ru \\ Roman Shmakov \\ FSBI «National Medical \\ Research Center For Obstetrics, \\ Gynecology \\ And Perinatology Named After \\ Academician V.I.Kulakov»》 \\ Ministry of Healthcare of the Russian \\ Federation, Moscow, Russia \\ r_shmakov@oparina4.ru
}

\author{
Oksana Vasilchenko \\ FSBI «National Medical \\ Research Center For Obstetrics, \\ Gynecology \\ And Perinatology Named After \\ Academician V.I.Kulakov»» \\ Ministry of Healthcare of the Russian \\ Federation, Moscow, Russia \\ vasilchenko-on@mail.ru \\ Vitaliy Chagovets \\ FSBI «National Medical \\ Research Center For Obstetrics, \\ Gynecology \\ And Perinatology Named After \\ Academician V.I.Kulakov»» \\ Ministry of Healthcare of the Russian \\ Federation, Moscow, Russia \\ vvchagovets@gmail.com \\ Gennadiy Sukhikh \\ FSBI «National Medical \\ Research Center For Obstetrics, \\ Gynecology \\ And Perinatology Named After \\ Academician V.I.Kulakov» \\ Ministry of Healthcare of the Russian \\ Federation, Moscow, Russia \\ g_sukhikh@oparina4.ru
}

\author{
Mariya Pirogova \\ FSBI «National Medical \\ Research Center For Obstetrics, \\ Gynecology \\ And Perinatology Named After \\ Academician V.I.Kulakov»» \\ Ministry of Healthcare of the Russian \\ Federation, Moscow, Russia \\ pirogovamariya@gmail.com \\ Larisa Ezhova \\ FSBI «National Medical \\ Research Center For Obstetrics, \\ Gynecology \\ And Perinatology Named After \\ Academician V.I.Kulakov»» \\ Ministry of Healthcare of the Russian \\ Federation, Moscow, Russia \\ 1_ezhova@oparina4.ru
}

\begin{abstract}
It is well-known that abnormal placental invasion is a condition characterized by pathological invasion of chorionic villi into the the basal layer of the uterus that is complicated by massive obstetric hemorrhage, blood transfusions, a need for hysterectomy and even mortality. The altered miRNA expression profiles were found by deep sequencing not only directly in the area of pathological invasion, but also in the outside distant placenta areas with more pronounced changes in the former case. An increase in the level of miR-21-5p, miR-25-3p, miR-92a-3p, miR-320a in maternal plasma may indicate abnormally invasive placenta and potentially be used in the early diagnosis and management of this condition.
\end{abstract}

Keywords - deep sequencing, RT-PCR, miRNA, placenta presentation, accreta, increta, percreta

\section{Motivation and aim}

Abnormal placental invasion is a condition characterized by pathological invasion of chorionic villi into the the basal layer of the uterus and as a result, it is complicated by massive obstetric hemorrhage, blood transfusions, a need for hysterectomy and even mortality [1]. Abnormal uterine scars after previous cesarean section especially in combination with placenta previa are thought to serve as a basis for defective decidualization and deep trophoblast invasion [2]. However, the molecular-biological profile of the placenta itself has not yet been studied in this pathology.
The aim of this study was to analyze and compare the miRNA expression profiles directly in the area of abnormal placental invasion (P-area) as well as in placenta areas outside this region $(\mathrm{N}$-area) in pregnant women with placenta accreta, increta and percreta in comparison with that in placenta presentation. Identified placental miRNAs were analyzed in maternal plasma as possible indicators of abnormally invasive placenta for further use as diagnostic and predictive tests.

\section{Methods}

69 pregnant women were taken into the study. Samples of a placenta tissue taken immediately after delivery were homogenized in a QIAzol Lysis Reagent, and the total RNA was isolated by an miRNeasy MicroKit and RNeasy MinElute Cleanup Kit (Qiagen, Hilden, Germany). miRNA from maternal blood plasma was isolated by an miRNeasy Serum/Plasma kit (Qiagen). Evaluation of the miRNA expression profile in placental tissue was performed using the NEBNext ${ }^{\circledR}$ Multiplex Small RNA Library Prep Set for Illumina ${ }^{\circledR}$ (Set11 and Set2, New England Biolab ${ }^{\circledR}$, Frankfurt am Main, Germany) for cDNA library synthesis and the NextSeq 500 platform (Illumina, San Diego, CA, USA) for sequencing. Differential expression analysis of the miRNA count data was performed with the DESeq2 package. The validation of deep sequencing data was carried out by reverse transcription coupled with polymerase chain reaction (RT$\mathrm{PCR}$ ) in real-time in a StepOnePlus ${ }^{\mathrm{TM}}$ thermocycler (Applied Biosystems, Foster City, CA, USA). 


\section{Results}

The altered miRNA expression profiles were detected not only directly in the area of pathological invasion (P-area), but also in the outside distant placenta areas (N-area). In the $\mathrm{N}$ sites, statistically significant changes in the expression level more than twice were found for 73 miRNAs when comparing the «increta group» with the «placenta presentation group» (for example, a 23-fold increase in the expression of miR-25$3 p, p=0.0002$; a 16.9-fold increase in the expression of miR92a-3p, $\mathrm{p}=0.0003$ ); for 67 miRNAs, when comparing the «percreta group» with the «placenta presentation group» (for example, a 15-fold increase in miR-25-3p expression, $p=7.8 \mathrm{e}-$ 5; a 16-fold increase in miR-92a-3p expression, $\mathrm{p}=0.0017$ ). In the P-sites, statistically significant changes in the expression level more than twice were found for 84 miRNAs when comparing the «increta group» with the «placenta presentation group» (for example, a 19-fold increase in the expression of miR-25-3p, $p=0.0002$; a 15 -fold increase in the expression of miR-92a-3p, $\mathrm{p}=0.0004$; a 2-fold increase in the expression of miR-320a, $p=0.02$ ); for 269 miRNAs, when comparing the «percreta group» with the «placenta presentation group» (for example, a 43-fold increase in miR-25-3p expression, $\mathrm{p}=5.7 \mathrm{e}-$ 40; a 37-fold increase in miR-92a-3p expression, $p=1.7 \mathrm{e}-44$; a 3.5 -fold increase in the expression of miR-320a, $p=1.78 \mathrm{e}-$
15 ; a 2-fold increase in the expression of miR-21-5p, $\mathrm{p}=0.029$ ). miRNAs specific for pathologic invasion of the placenta were validated in blood plasma by the quantitative RT-PCR on an independent cohort of pregnant women. There were 8.8- and 10-fold increases in miR-21-5p level ( $\mathrm{p}=0.0003$ and $\mathrm{p}=0.0029$, respectively), 3 - and 5-fold increases in miR$25-3 p$ level $(\mathrm{p}=0.05$ and $\mathrm{p}=0.04$, respectively), 1.35 - and 1.9fold increases in miR-92a-3p level $(p=0.033$ and $p=0.001$, respectively), 1.9- and 2.2-fold increases in miR-320a ( $p=0.005$ and $p=0.001$, respectively) in the «increta group» and «accreta group», respectively, relative to the «placenta previa group» without an uterine scar. An increase in the level of miR-21-5p, miR-25-3p, miR-92a-3p, miR-320a in maternal plasma may indicate abnormally invasive placenta.

\section{ACKNOWLEDGMENT}

Supported by the State assignment of the Ministry of Healthcare of the Russian Federation.

\section{REFERENCES}

[1] L. Say et al. Global causes of maternal death: A WHO systematic analysis. Lancet Glob. Heal. 2014.Vol. 2, № 6, P. 323-333.

[2] Tantbirojn P, Crum CP, Parast MM. Pathophysiology of placenta creta: The role of decidua and extravillous trophoblast. Placenta 2008; 29:639-645 